\title{
Variations
}

Variations

Revue internationale de théorie critique

$13 / 14 \mid 2010$

Le choix du petit

\section{Imitation, proximité, sensibilité}

De l'échelle tribale du communisme

Jean-François Gava

\section{(2) OpenEdition}

Journals

Édition électronique

URL : http://journals.openedition.org/variations/178

DOI : $10.4000 /$ variations. 178

ISSN : 1968-3960

Éditeur

Les amis de Variations

Édition imprimée

Date de publication : 31 mars 2010

Référence électronique

Jean-François Gava, «Imitation, proximité, sensibilité », Variations [En ligne], 13/14 | 2010, mis en ligne le 01 février 2012, consulté le 20 avril 2019. URL : http://journals.openedition.org/variations/178 ;

DOI : 10.4000/variations. 178

Ce document a été généré automatiquement le 20 avril 2019.

Les ami•e•s de Variations 


\title{
Imitation, proximité, sensibilité
}

\author{
De l'échelle tribale du communisme
}

Jean-François Gava

\section{NOTE DE L'ÉDITEUR}

Première publication sur www.theoriecritique.com, « Le choix du petit », Printemps 2010, pp. 66-73

1 Le communisme est la philosophie de la société directe. Par conséquent, son échelle naturelle est le village - que celui-ci s'entende au sens traditionnel ou qu'il s'importe en contexte urbain. En outre, un monde de communes nous siérait à ravir, mais la perspective n'est plus désormais celle du ralliement de masse, plutôt celle d'îlots de survie. Le communisme en petits comités : voyez-vous ça! L'on entend d'ici fuser la raillerie des éveilleurs de conscience purs et durs, ceux-là mêmes qui n'oignaient les jeunes incendiaires des banlieues françaises que de leur mépris, en novembre 2005. Aurions-nous ici, diront-ils, de nouveaux gnostiques, les adeptes d'une vérité à laquelle seuls les initiés accéderaient? L'émancipation n'est-elle pas au contraire fondamentalement exotérique? Ne doit-elle pas entraîner dans son sillage le plus grand nombre? Puis-je être libre seul, alors que personne n'est libre tant que tous ne le sont pas? Que la commune soit affaire de misanthropes est une absurdité, même si elle ménage en son sein toute latitude aux solitaires; mais qu'elle soit affaire de masses est une absurdité non moins grande.

D’autres nous auraient reproché notre prêchi-prêcha de prosélytes, nous en fussionsnous tenus à la version religieuse jadis : 'allez enseigner les nations et convertissez les masses !'. L'animosité des impuissants, qui ne sont pas le contraire des gens de pouvoir, est, comme on le voit, protéiforme. Si le cadet de nos soucis est bien de partir sur les routes pour annoncer la bonne nouvelle, c'est qu'une vie prêche d'elle-même en sa faveur (ou en sa défaveur). Veritas index sui, disait Spinoza. Nous ne cherchons à convaincre personne. La meilleure stratégie de séduction en faveur d'une puissance de vie est l'exercice de cette puissance lui-même. Mais que les fortes têtes libérales ne s'imaginent pas trouver en 
nous à si bon compte des alliés conscients pour autant. Le communalisme est moins le fait d'un club d'opinion que la caractéristique du vivant en général ; la vie parle communal comme Monsieur Jourdain prosaïque : sans le savoir. C'est pourquoi le vivre communal est le fait au moins virtuel de tous : la commune est l'inconscient, ce dernier fût-il en voie de disparition. Rien ne réjouit davantage les communaux que l'irruption éruptive d'une autre commune, même aux antipodes. Ils ne se croiront pas obligés de faire 'commune' impossible avec ces derniers pour autant. La société planétaire est celle du capital; les 'multitudinaires' qui voudraient en changer le signe sans en changer l'échelle nous font croire à cette société-ci : froide, lointaine, extérieure. Ils ne font que reprendre à leur compte l'imbécillité congénitale du marxisme, que Marx vouait déjà aux gémonies. Merveilleuse globalisation, disent-ils! Pourvu que nous nous en appropriions les fruits ! Fadaises délétères que tout cela; le socialisme tant vitupéré par ces mêmes globalistes trouve en réalité chez ces derniers ses habits neufs, hi-tech, immatériels, tout ce qu'on voudra de trendy et pour tout dire de métropolitain.

3 Nous ne croyons de toute façon pas à une quelconque contagion qui soit assez massive pour interrompre délibérément, ou même éruptivement, le cours de cette forme pathologique de civilisation, qui ne s'interrompra que de l'épuisement de son combustible; même si le socialisme était souhaitable, il serait impossible. Aucun projet hétérogène n'est désormais capable de gripper le processus, qui touche de toute façon à sa fin. Non seulement le mouvement ouvrier a gâché la seule occasion sérieuse qui se soit jamais présentée à l'échelle globale (la première guerre mondiale), mais il aurait fort probablement voué sa victoire à la poursuite de la même affaire par d'autres moyens. Aujourd'hui, il suffit, pour prendre la mesure de la servitude, d'imaginer les conséquences que ne manquerait pas d'entraîner la moindre mesure thérapeutique de gouvernement vouée à l'émancipation ${ }^{1}$ : elle rencontrerait certainement une résistance massive comme à une mesure 'liberticide' et pourquoi pas fasciste. Il faut nous rendre à l'évidence de ce qu'aujourd'hui, une émancipation administrée, si elle était possible, comme Krahl le croyait encore ${ }^{2}$ , serait sans aucun doute 'reçue' comme du fascisme pur et simple par le plus grand nombre. Telle est la plus grande servitude que la plus grande liberté elle-même apparaitrait aux serfs comme la plus grande servitude.

Prosélytisme impossible, socialisme de toute façon peu souhaitable. L'issue est désormais communale et locale ; elle consiste à consolider des noyaux sociaux capables de survivre à l'effondrement systémique, de persister et perdurer à travers lui. Le communal, c'est le communisme à petite échelle, parce qu'il n'y en a pas d'autre. Il est aussi insensé de faire du capitalisme à échelle humaine que du communisme à échelle capitaliste. La société globale est la société capitaliste, et aucune autre. L'industrie capitaliste, c'est le pharaonisme; or la grande industrie est inappropriable. On ne se réapproprie pas le constitutivement impropre. Le gigantisme machinique engendré par la subsomption réelle est fondé sur les cadavres des aptitudes, outre d'être fondé sur les cadavres des cultures, des formes de vie en général, ainsi forcément que de leurs suppôts, les individus vivants. Qui ira se réapproprier cette gigantesque nécropole de la créativité qu'est le bureau-usine global ? On ne peut se réapproprier que de l'exproprié au sens patrimonial : le machinisme est fonction directe de l'expropriation technique; le capitalisme est le capital s'emparant des techniques pour les détruire. Quel sens pourrait avoir la réappropriation de l'instrument de destruction de toute propriété, entendue comme ce qui s'oppose à l'impropre? 
5 La levée de boucliers ne se fait pas attendre du côté d'un marxisme désireux de s'émanciper du Marxisme et rendant hommage malgré lui à cette civilisation machiniste, en protestant d'un bon sens qui voile mal la panique: "cette entropie politique nous demande de quitter nos cabinets, au bénéfice des toilettes sèches.» (A. Neumann, Conscience de casse, p. 110), et de poursuivre : « aucune société ne s'est jamais effondrée à cause du seul problème environnemental » (ibid.). Cela revient pratiquement à ne pas distinguer entre migration cyclique/saisonnière des peuplades primitives en direction de territoires davantage pourvus en ressources alimentaires, d'une part, et exploitation intensive du support naturel de 'la' nature humaine en régime capitaliste, incompatible avec la reconstitution dudit support, de l'autre. En gros, la nouvelle École de Francfort nous ramène aux années 60 , où on la dirait restée, avec son marxisme sans doute postprolétarien, mais certes pas post-industriel : «la révolution (...) prend naissance à un stade avancé de la société industrielle» (Marcuse, "Réexamen du concept de révolution ", in Diogène n $\left.^{\circ} 64,1968\right)^{3}$. Ferait-on l'impasse sur la mise en garde formulée il y a plus de 35 ans par Schumacher dans l'incipit de son opus princeps : « une des erreurs fatales de notre temps est de croire résolu le problème de la production.» (Small is beautiful. Une société à la mesure de l'homme [1973], Seuil, 1978, p. 13)?

6 Serions-nous donc technophobes? Tel est le qualificatif que se voit inévitablement décerner toute critique un peu scrupuleuse du capitalisme, dans un jugement où culmine l'incompréhension aussi bien de la notion de technique que de la nature même du capitalisme. Méconnaissant les 'incontestables' avancées d'une civilisation qui a, en réalité, consciencieusement et méthodiquement broyé en trois siècles tout ce que l'humanité avait accumulé de technique depuis le néolithique, nous nous rendrions coupables de technophobie, alors que notre philosophie pourrait se résumer à un naturalisme techniciste. Aimer la machinerie qui, après avoir ravagé nos terres et nos corps, envahit nos paniers de biens sous forme de véhicules automobiles, d'électroménagers et d'électronique domestique, cela s'appellerait de la technophilie : voire !

7 Entendons évidemment par industrie : capitalisme ${ }^{4}$. C'est le sens le plus commun, même si sous la plume de Hegel, l'ordre industrieux était encore tout sauf post-artisanal; industrie signifiait tout bonnement le règne de l'effort. Il faudrait dire donc, plus encore qu'industrie: machinisme, pour éviter toute coloration technique ou artisanale. Le machinisme se substitue à la technique en l'extrayant des corps ouvriers; il renverse ainsi le rapport de subordination jadis de l'outil à l'ouvrier, non sans en transformer les termes qui deviennent : machine et travailleur (abstrait).

8 Il est étonnant qu'un penseur au moins à moitié perspicace tel que Stiegler (noyant pour l'autre moitié, dans de virtuoses gerbes lexicales, le poisson d'une inanité progressant en raison inverse de leur intelligibilité) situe le déclin des savoir-vivre à l'âge, qu'il qualifie de consumériste, du XXe siècle. Auparavant, le capital n'aurait entamé que les savoir-faire ouvriers, c'est-à-dire artisanaux, et paysans. Tout se passe comme s'il avait fallu attendre le remplissage administré du vide créé dans les corps par la transformation des producteurs en force de travail pour que ce vide soit, alors qu'il ne faisait ainsi que se manifester. Quelle curieuse hypostase! D'un côté donc, les savoir-faire, de l'autre, les savoir-vivre (user de, consommer) et ce, alors que notre auteur stigmatise lui-même ce déni de coappartenance, en s'appuyant sur Marx en personne, celui de la fameuse Introduction à la critique de l'économie politique de $1857^{5}$, écrivant que « cette production qui s'identifie à la consommation est une deuxième production, issue de la destruction du premier produit. Dans la première, le producteur s'objectivait; dans la seconde au contraire, c'est l'objet 
qu'il a créé qui se personnifie » (in Contribution à la critique de l'économie politique, ES, 1968, pp. 155-6 ; l'édition Altiplano 2008 de l'Introduction étant plus accessible, on y trouvera ce texte aux pp. 40-1). La prolétarisation est bien l'effet de la vidange des corps techniciens, que ces techniques se déploient activement plutôt du côté du pôle usager (consomptif) ou plutôt du côté du pôle productif.

Ceux-là mêmes qui font le diagnostic de la prolétarisation générale (technique et non seulement patrimoniale) se bornent à vouloir sauver le seul consommateur et ses savoirvivre, et avec eux le capitalisme, qu'il faudrait tirer, allons donc, la vieille antienne !, de son ornière financière (ce méchant capitalisme financier qui a si bon dos) pour le faire accéder à un nouvel âge de l'esprit : et de partir de vrombissants appels à des politiques publiques d'investissements à long terme en vue de commuer les nouvelles techniques et technologies de l'esprit en hypomnemata ou grammatisations (sic) en milieux associés (sic) d'où l'esprit sortirait émancipé au lieu de finir abruti, comme c'est ce qui nous attend. Ces rodomontades à dormir debout, on les trouve sous la plume de 'philosophes' pressés, obsédés de conversion et surtout de cooptation étatiques et débondant des torrents de logomachie ronflante, qui vont jusqu'à brandir l'étendard de l'ars industrialis, ignorant manifestement tout du pléonasme, et davantage encore de ce que pourrait être une industria artificialis. 'Surtout pas de décroissance !', assènent ces futurs ministres de la recherche.

'Et les progrès de la science !' aboient les ilotes. 'Rends-nous tes lunettes !' intiment les scientolâtres. Un peu comme si, entendant maugréer à cause de leurs maudites prothèses des manchots tout juste revenus d'une guerre juteuse où il les aurait envoyés se faire mutiler, le Grand Conseil de l'Industrie leur crachait au visage: 'rends-nous tes béquilles !'. Ce chantage ignominieux remonte à Freud au plus tard, qui s'en moquait déjà en 1929: «N'est-ce donc pas un gain de plaisir positif, un surcroît sans équivoque de sentiment de bonheur, que de pouvoir entendre aussi souvent qu'il me plaît la voix de l'enfant qui vit loin de moi, à des centaines de kilomètres de distance (...) ? Cela ne signifie-t-il rien que la médecine ait réussi (...) à prolonger d'un nombre considérable d'années la durée de vie moyenne de l'homme de la culture? ", ironisait-il, pour répondre aussitôt: "S'il n'y avait pas de chemin de fer pour surmonter les distances, l'enfant n'aurait jamais quitté sa ville natale, on n'aurait pas besoin de téléphone pour entendre sa voix (...) Et enfin, à quoi bon une longue vie, si elle est pénible, pauvre en joies et si chargée de souffrance que nous ne pouvons accueillir la mort qu'en rédemptrice?» ( Malaise dans la culture, coll. Quadrige, 1995, pp. 31-2) ». À sa suite, le Groupe Oblomoff, le CNRS (Coordination Nationale de Répression du Scientisme) et bien d'autres encore ont tout dit à ce sujet.

11 Quant aux béquilles dont il s'agit de se débarrasser, l'image est quelque peu bancale (c'est le cas de le dire), car les membres (re)tranchés ne repoussent pas ; il faut pourtant penser la nouvelle civilisation sur le modèle de la réhabilitation des grands lésés.

Quelle communauté problématique entre industrie moderne et petitesse de la production autonome? C'est que l'industrie moderne a partie liée avec les grands ensembles, pour emprunter l'expression à Patrick Marcolini. Marx nous a enseigné la loi de concentration du capital (grands ensembles), qui va de pair avec celle de la centralisation (de la propriété). La constitution post-fordiste de grappes 'd'accumulation dynamique', redéploiement par lequel les grands ensembles procèdent à l'externalisation de bon nombre de fonctions, biens et services B2B (Business To Business), déléguées à un chapelet de PME, ne doit pas faire illusion. Ce petit-là n'est pas le nôtre, et son 'artisanat', ses 
compétences de niche, servent le grand et le lointain. Le petit de l'artisan, du technicien au sens propre et véritable, pas celui qui sert la machine, qui n'a connaissance que de cette machine qui l'exproprie de la technicité de son être, ne sert que le petit. La production non-capitaliste est seule à la mesure de l'infini, de l'autonomie communale, parce que l'outil est subordonné à l'ouvrier (ici encore entendu au sens propre et véritable) plutôt que le prolétaire n'est subordonné à la machine. Le petit de l'outil offre ce dernier à la fluidité infinie de la maîtrise technique individuelle. De même, le petit de la communauté offre prise elle aussi aux singularités, elle n'est pas indifférente à ces singularités dont l'individu est l'élément fondamental. Mais la division des tâches est le résultat de la communauté, et non la conséquence de l'insuffisance individuelle. L'homme, forcément individu, est plus fort regroupé, rabat toute l'anthropologie moderne ; mais c'est là concevoir le commun en pure extériorité par rapport à l'individu. Le commun fournit bien plutôt d'emblée à l'individu l'image extérieure, comme monde organisé et forcément partagé, de cette infinité dont il est le siège conscient. L'individu est l'unité minimale de diffraction infinie d'une vie commune qui l'enveloppe.

\section{NOTES}

1. Émancipation non pas du (ablatif) lien social, celle qu'accomplit le capitalisme, mais du lien de servilité et de mépris, qui empêche tous les liens spontanés et que le capitalisme porte à son état de pureté pour ainsi dire chimique.

2. «Die Massen sind in der autoritären Leistungsgesellschaft von Erziehung, Manipulation und exekutiver Indoktrinierung so sehr auf Autoritäten fixiert, dass sie zunächst für ihre Aufklärung selber Autoritäten - und zwar solche, die sich als kritische Autoritäten begreifen - nötig haben » (http://www.krahlstudien.de).

3. Mais écoutons plutôt à ce sujet l'une des références cardinales de cette nouvelle École : «ce serait certainement un erreur de reprendre l'idée romantique d'une décentralisation complète des organisations communautaires et des sites de production (...) qui ne saurait s'appliquer sans autre médiation dans les conditions des sociétés industrielles complexes et de leurs États prenant appui sur de vastes territoires » (Negt, 'Pas de démocratie sans socialisme, pas de socialisme sans démocratie' [1978], in L'espace public oppositionnel, Payot, 2007, p. 36). Tout semble se passer ici comme si la société industrielle allait de soi. On se demande ce qu'il est advenu de la critique de l'économie politique!

4. Mais 'industrie' aujourd'hui présente l'avantage de recouvrir aussi bien les versions orientales (ou 'socialistes') qu'occidentales du capitalisme, cette catastrophe survenue dans l'histoire de la biosphère il y a quelques siècles à peine du seul fait de notre espèce. Laps de temps sans doute dérisoire à l'échelle de l'histoire de la vie elle-même, mais qui a suffi aux hommes pour conduire cette dernière tout près de l'exténuation.

5. Restée impubliée du temps de Marx, mais mentionnée par celui-ci dans la préface de 1859 à la Contribution et jointe à cette dernière dans l'édition française 1968 de ladite Contribution. 


\section{AUTEUR}

\section{JEAN-FRANÇOIS GAVA}

Docteur en philosophie, Chercheur indépendant, CREALA. 